\title{
Devenir du marquage sexuel du noyau dans les transplants hétéro-sexuels de muqueuse vésicale
}

\author{
A. Caratzali \\ (avec la collaboration technique de E. Ghidus-Marinesco et D. Marin)
}

(Bucarest)

Dans des travaux antérieurs, nous avons constaté que la chromatine sexuelle des granulocytes neutrophiles est susceptible d'importantes variations quantitatives sous l'influence de diverses actions hormonales. Ainsi, au cours de la seconde phase du cycle menstruel ( I) des premiers mois de la grossesse (2) et du stress expérimental, (3) le nombre des corpuscules sexuels des granulocytes neutrophiles, tant pédiculés que sessiles, augmente de façon significative. Ceci vient à l'appui des constatations antérieures aux nôtres faites par Romatowski, Tolksdorf et Wiedemann (4) concernant le grand nombre des "drumsticks » observé chez les fillettes prématurées.

Or, il n'en est pas de même des observations faites sur la chromatine sexuelle des cellules somatiques autres que celle des granulocytes. De nombreux auteurs $(5,6,7,8)$ ont en effet constaté que la chromatine sexuelle de ces cellules reste indifférente à l'égard de l'impregnation hormonale de l'organisme, normale ou produite par voie expérimentale.

Pour contribuer a l'éclaircissement du problème, nous avons effectué une série de transplants de muqueuse vésicale chez le chien, d'un sexe à l'autre. On sait qu'un lambeau de muqueuse vésicale inséré sous une aponévrose musculaire provoque la formation d'un kyste. Au bout d'une dizaine de jours, ce kyste induit la transformation des tissus avoisinants en cartilage, puis en os.

La question que nous nous sommes posée a été de savoir si, dans ces conditions, le caryotype sexuel des cellules transplantées conservait les caractéristiques du donneur ou bien se modifiait sous l'influence de l'hôte, en prenant la marque du sexe du receveur.

Technique. Un lambeau de muqueuse vésicale était filatovisé pendant quatre jours à la glacière, dans une solution faible de pénicilline. Ce laps de temps passé, le lambeau était inséré sous l'aponévrose du grand droit abdominal d'un chien ayant le sexe opposé au sexe de l'animal donneur. Au bout de 9 jours, le kyste était extirpé et examiné au point de voe cytologique. L'examen cytologique aussi bien du lambeau, avant et après filatovisation, que de la formation kystique a été fait sur des frottis et sur des coupes. Les frottis ont été fixés au mélange de Papanicolaou. Pour 
les coupes, la fixation a été faite aux liquides d'Orth et de Helly. Des colorations parallèles ont été faites à l'hématoxyline de Harris et au réactif de Feulgen. Chaque lecture a comporté le dénombrement de 250 cellules. Les diverses méthodes employées ont mené à des résultats concordants. Ajoutons que le diagnostic de caryotype sexuel a été plus facile sur les frottis que sur les coupes. En outre, le nombre des cellules à marquage sexucl a été plus élevé sur les frottis (près de $50 \%$ chez les femelles, 3 à $4 \%$ chez les mãles) que sur les coupes (environ $35 \%$ chez les femelles et $2 \%$ chez les mâles).

Nous avons observé que le marquage sexuel a été le plus évident sur les grandes cellules épitheliales, à noyau grand et clair, ainsi que l'a constaté Danon (9) sur les coupes de peau. Dans nos observations, la grande majorité des noyaux porte un seul amas chromatinien sexuel, indifféremment du nombre des nucléoles.

Résultats et conclusions. Dans tous les cas observés, les cellules épithéliales de la néoformation kystique appartiennent au caryotype sexuel de la muqueuse vésicale du donneur. Elles tranchent d'une façon net sur les cellules des tissus du receveur à caryotype sexuel différent. Il ne semble donc pas avoir d'interaction entre tissus du donneur et tissus du receveur: les cellules de ces tissus conservent leurs traits sexuels génétiques. Remarquons, en passant, que la filatovisation garde le caryotype sexuel propre au tissu réfrigéré.

Ainsi donc, les humeurs et les tissus de l'hôte ne paraissent pas avoir d'influence sur le caractère sexuel génétique du noyau des cellules transplantées, tout au moins dans le cas de la muqueuse vésicale. Ces constatations vont à l'encontre des observations faites sur les variations physiologiques des corpuscules sexuels des granulocytes. Ces faits semblent confirmer l'opinion de quelques auteurs, dont Ashley, (I0) Sander, (I I) Sahleanu (I2) à savoir que la chromatine constitutive des "drumsticks" de Davidson (I3) d'une part et, d'autre part, la chromatine de Barr, (I4), (I5) soit sous forme de satellite nucléolaire, soit sous forme de nodule plus au moins adhérent à la membrane nucléaire - ne seraient pas des formations homologues: seule la chromatine de Barr traduirait le sexe génétique.

\section{Sommaire}

Chez le chien, le transplant de muqueuse vésicale à un animal de sexe opposé conserve, lors de la formation du kyste, son propre caryotype sexuel. Ceci semble démontrer - à l'opposé de ce qui se passe pour les corpuscules sexuels leucocytaires - que le caryotype sexuel de certaines cellules ne subit pas des modifications sous l'influence de l'impregnation hormonale. 


\section{Bibliographie}

I. Caratzali A., Phleps A., Turpin R.: Bull. de l'Acad. Nat. de Méd. 1957, I4I, 22, 496.

2. Caratzali A., Ghidus-Marinesco E., Phleps A.: Sess. scient. festive de l'Institut Médico-Pharmaceutique de Bucarest, séance du 28 déc. 1957.

3. Carazali A., Holban R., Phleps A.: Studii si cercetari de Endocr., 1959, Nr. 2 ('sous presse).

4. Romatovski H., Tolksdorf M., Wiedemann H. R.: Klin. Wochschr, 1955, 33, 9 i 1.

5. Graham M. A.: Anat. Rec. 1954, i ig, 469.

6. Grumbach M. M., Blanc W. A., Engle E. T.: J. of Clin. Endocr. a. Metab., 1957, i 7, 3, 454.

7. Wrtschi E., Nelson W. O., Segal S. V.: J. of clin. Endocr. a Metab., 1957, 17, 3, 737.

8. Danila Muster A., Bal.aceanu Stolnici M.: Studii si cercet. de Endocr., i959 (sous presse).

9. Danon M., SAGhs L.: Lancet, 1957, ii, 20.

10. Ashley D. V. B., Jones C. H.: Lancet, I958, i, 240 .

I I. Sander E.: Communication personnelle.

i2. Maicanescu M., Sahleanu V., Ionescu B.: Probleme de sexologie masculina, i958, Bucaresti, Edit. Med.

13. Davidson W. M., Robertson Smith D.: Brit. Med. J., 1954, ii, 6.

I4. Barr M. L., Bertram E. G.: Nature, Lond., 1949, I63, 676.

I 5. Moore K. I.., Barr M. L.: Lancet, 1955, ii, 57. 


\section{RIASSUNTO}

Il trapianto, nel cane, della mucosa vescicale ad un animale di sesso opposto conserva, alla formazione della ciste, il proprio cariotipo sessuale. Ciò sembra dimostrare - all'opposto di quanto si verifica per i corpuscoli sessuali leucocitari - che il cariotipo sessuale di determinate cellule non subisce modificazioni sotto l'azione dell'impregnazione ormonale.

\section{SUMMARY}

Transplants of vesical mucous tissue in the dog to an animal of opposite sex keep their own sex karyotype.

This seems to prove-to the contrary of what takes place for leucocytic sex corpuscles -that the sex karyotype of some cells does not undergo alterations under the action of hormone impregnation.

\section{ZUSAMMENFASSUNG}

Das Implantat von Blasenschleimhaut auf ein Tier des entgegengesetzten Geschlechtes beim Hund behält bei Zystenbildung den dem Geschlecht eigenen Karyotyp. Das scheint zı! beweisen - entgegen dem, was sich bei den leukozytären Geschlechtskörperchen ereignet dass der Geschlechtskaryoryp bestimmter Ze'len unter der Wirkung der Hormonimprägnierung keine Veränderungen durchmacht. 